\title{
Soyons égoïstes, soyez généreux. Le Cycle du développement à l'OMC
}

\section{Tancrède Voituriez}

\section{(2) OpenEdition}

1 Journals

\section{Édition électronique}

URL : http://journals.openedition.org/economierurale/609

DOI : 10.4000/economierurale.609

ISSN : 2105-2581

\section{Éditeur}

Société Française d'Économie Rurale (SFER)

\section{Édition imprimée}

Date de publication : 1 mars 2006

Pagination : 72-80

ISSN : 0013-0559

\section{Référence électronique}

Tancrède Voituriez, «Soyons égoïstes, soyez généreux. Le Cycle du développement à l'OMC »,

Économie rurale [En ligne], 291 | Janvier-février 2006, mis en ligne le 01 mai 2008, consulté le 19 avril 2019. URL : http://journals.openedition.org/economierurale/609; DOI : 10.4000/economierurale.609

Ce document a été généré automatiquement le 19 avril 2019

(c) Tous droits réservés 


\title{
Soyons égoïstes, soyez généreux. Le Cycle du développement à l'OMC
}

\author{
Tancrède Voituriez
}

1 La conférence ministérielle de l'Organisation mondiale du commerce (OMC) qui s'est tenue à Hong-Kong du 13 au 18 décembre 2005 s'est conclue par une déclaration dont l'existence, plus que le contenu lui-même, est présentée comme le principal succès des six jours de négociation. Succès modeste à la mesure des ambitions tout aussi modestes prêtées à la rencontre qui ne visait qu'à remettre l'agenda de Doha sur les rails et repousse à un an la conciliation d'intérêts contradictoires subsistant entre pays membres de l'Organisation.

2 Symptomatique de la modestie des ambitions, l'élimination de toute forme de subventions aux exportations agricoles à l'horizon 2013 a été présentée dans les quotidiens, le lendemain du terme de la conférence, comme la mesure emblématique du progrès des négociations vers une libéralisation effective des échanges. Des grincheux perspicaces, comme The Economist,eurent cependant tôt fait d'observer que l'élimination des subventions à l'exportation surviendrait mécaniquement avant 2013 en raison des réformes des politiques agricoles internes - singulièrement en Europe, principalement visée par la mesure - conduites dans les pays concernés. Même si ces réformes ont été, pour une large part, induites par le précédent accord signé à l'OMC (l'Accord agricole de Marrakech, signé en 1994), nul besoin de déclaration ministérielle de Hong-Kong pour obtenir des subventions aux exportations à un niveau proche de zéro sur un horizon aussi éloigné. Si l'on se souvient qu'un accord multilatéral est justifié dans la mesure où chaque pays se trouve, après accord, dans une "meilleure » situation (quel qu'en soit l'aune) qu'avant accord d'une part, et que cette «meilleure» situation d'autre part n'aurait pu être atteinte sans l'accord considéré, alors la déclaration de Hong-Kong, si l'on s'en tient à la mesure phare qu'est la suppression des subventions à l'exportation, n'est pas la refondation éclatante du multilatéralisme que l'on a pu annoncer.

3 La question qui se pose alors, ouverte après l'échec des ministérielles de Seattle et Cancun et à laquelle la déclaration de Hong-Kong ne répond pas, est de savoir ce qui, dans les 
engagements de Doha visant à faire de l'actuel cycle de négociation un généreux « cycle du développement ", produit des inconciliabilités à ce point féroces que les principes de l'Organisation, éprouvés depuis l'instauration du GATT en 1947, qui sont, pour les premiers d'entre eux, la réciprocité et la non-discrimination (principe de la nation la plus favorisée et du traitement national) se trouvent pris en défaut, de même que le corps de doctrine économique sur lequel ce premier accord, puis les suivants, se sont appuyés.

\section{Guerre, lobbys et représailles}

Sur quel corps de doctrine le GATT a-t-il donc été élaboré ? Une réponse, que l'on peut lire ou entendre, est celle des avantages comparatifs de Ricardo. Or, cette réponse est très largement erronée. Lorsque Stanislaw Ulam, un des pères de la bombe $\mathrm{H}$, avait mis au défi quiconque de trouver une seule théorie dans toutes les sciences sociales qui soit à la fois vraie et non triviale, Paul Samuelson avait répondu en citant la théorie des avantages comparatifs de Ricardo. Qu'elle soit vraie, d'un point de vue logique, n'est pas à démontrer à un mathématicien, avait répondu Samuelson ; et elle n'est pas triviale, comme l'attestent les milliers d'hommes intelligents et importants qui n'ont jamais su la saisir ou tout simplement la croire après qu'on la leur eut expliquée. On se souvient aussi que cette même loi, garantissant à chaque petits pays d'accroître sa richesse s'il procède à une libéralisation de son commerce quoi que fassent les autres pays, soulignait l'apparente incongruité des accords multilatéraux de libéralisation commerciale; ces derniers en effet contraignant les pays à poursuivre leur propre bonheur. Pourquoi diable les pays se doteraient-ils de règles communes les obligeant à libéraliser leur commerce, quand libéraliser de manière unilatérale apporte prospérité et richesse? La littérature économique, exposant la rationalité et l'histoire du GATT, apporte trois réponses à cette question :

5 La première réponse est d'éviter le protectionnisme qui dans les années trente a (ou aurait) conduit à la guerre. Insistant sur les effets en cascade que les tarifs SmoothHawley inaugurèrent après leur vote au Congrès américain en 1930, puis sur les conséquences économiques et politiques qu'ils infligèrent ${ }^{1}$, un auteur comme Pastor (1983) énonce cette idée partagée avec retard par l'administration américaine selon laquelle «les droits de douane Smooth-Hawley de 1930 sont au commerce ce que les accords de Munich 1938 sont à la paix». Cordell Hull, secrétaire d'État de Roosevelt pendant 12 ans, partage semblable prévention. "Le commerce sans frein ", écrit-il, dans ses mémoires en 1948, "s'accorde à la paix, les droits de douane élevés, les barrières commerciales et une concurrence économique injuste s'accordent à la guerre». Une des premières mesures de l'Administration Roosevelt après 1933 sera d'essayer d'accroître les débouchés extérieurs, et de négocier une baisse des droits de douane sur le vieux continent, en échange d'un accès accru des produits étrangers au marché américain. Le Reciprocal Trade Agreement Act (RTAA) de 1934 marque la naissance politique du principe de réciprocité, pilier du GATT et de l'OMC, grâce auquel le poids des lobbys protectionnistes peut être surmonté par l'assurance donnée aux lobbys exportateurs d'un accès réciproque aux marchés extérieurs. Elle est, par ailleurs, conforme à la doctrine démocrate d'avant-guerre en matière de commerce, doctrine volontiers libérale, au sens français du terme, qui, contre les intérêts des industries du nord, soutenues par les Républicains, n'eut depuis 1860 que deux occasions de modifier les tarifs, en 1894 et 1913, et dans les deux cas, nettement à la baisse. Le RTAA contiendra à grand-peine la surenchère protectionniste et le repli 
impérial de la France, de la Grande-Bretagne et de l'Allemagne. La guerre terminée, la leçon qu'en tire Hull est que le commerce désentravé «offre une chance raisonnable d'assurer la paix ».

6 La seconde réponse est d'apporter aux gouvernements un contrepoids face aux lobbys protectionnistes de leurs propres pays. Le principe de réciprocité, dont on a vu que le RTAA marquait la première application, peut être lu dans deux sens. Une première lecture, sans doute la plus conventionnelle, le décrit comme un moyen d'obtenir des parts de marché extérieur. Une seconde lecture, tout aussi exacte, renverse la perspective et place la finalité du principe de réciprocité dans l'économie domestique elle-même, grâce à la circonscription des lobbys prompts à acheter la protection du Congrès. La relation entre ces deux objectifs est en réalité circulaire, l'ouverture du marché intérieur étant, selon le point de vue, la cause et la conséquence d'un accès accru aux marchés extérieurs. En prolongeant cette conception de la justification d'un accord multilatéral, Hoekman et Kostecki (2001) dans leur ouvrage de référence sur l'économie politique de l'OMC, voient dans l'Organisation un moyen de soustraire les gouvernements à l'influence de leurs propres démons (protectionnistes s'entend). Ainsi affirment-ils, utilisant une comparaison due à Roessler (1985) : «Les règles et principes de l'OMC contraignent la liberté des gouvernements dans l'usage d'instruments particuliers de politique commerciale. Ces règles et principes influencent l'équilibre politique entre les groupes d'intérêt protectionnistes et ceux plus favorables à l'ouverture des marchés. [...] L'OMC est, d'une certaine manière, analogue à un mât auquel les gouvernements décident de s'attacher afin d'échapper aux chants de sirènes entonnés par les différents groupes de pression.»

7 La troisième réponse est de mettre un terme, par le jeu des concessions réciproques dans l'accès au marché, aux droits de douane que les grands pays importateurs instaurent afin, soit de déprimer les cours mondiaux et donc de réduire à leur avantage leurs dépenses d'importation, soit d'infliger des représailles commerciales à un pays partenaire qui, chez lui, en aurait fait de même. Ce dernier cas de figure échappe à la théorie Ricardienne puisqu'un grand pays, parce qu'il peut influencer les prix, a tout intérêt à mettre en place une protection non nulle afin de manipuler en sa faveur les cours mondiaux. Il n'en va pas de même lorsqu'existent plusieurs grands pays. Les représailles, escalades tarifaires et guerres commerciales conduisent alors à une perte nette pour tous sans que chacun n'ait intérêt, seul, à retrouver une situation moins protégée (Bagwell et Steiger, 1999). Selon cette réponse apportée à une situation bien connue dite de «dilemme du prisonnier ", le GATT est d'abord une affaire de grands pays exportateurs et importateurs, d'une part capables de manipuler les termes de l'échange au risque de s'y enferrer, de l'autre, incapables de renoncer à leurs droits de douane en dépit de leur excès, à moins que ne soit obtenue l'assurance que les pays partenaires, eux aussi grands pays et surprotégés, concèdent à l'identique une ouverture accrue de leurs marchés. Les négociations agricoles, entamées lors du cycle d'Uruguay, en apportent une illustration graphique convaincante. L'émergence de l'Europe comme grand pays dans ce secteur, contestant la prééminence des États-Unis, s'est traduite au tournant des années 1970 et 1980 par une débauche de différends et de protections conduisant à l'inclusion de l'agriculture dans le cycle de libéralisation au moment même où l'Europe rejoignait les États-Unis en termes de parts de marché mondial (graphique ci-après).

8 Le GATT selon ces trois réponses permet à chaque pays de se défaire de ses mauvaises politiques, qu'elles procèdent d'une logique guerrière, du jeu de lobbys ou de représailles. 
Chaque pays ne peut s'en défaire tout seul. Le GATT est un accord multilatéral pleinement justifié.

Figure 1. Exportations de produits alimentaires

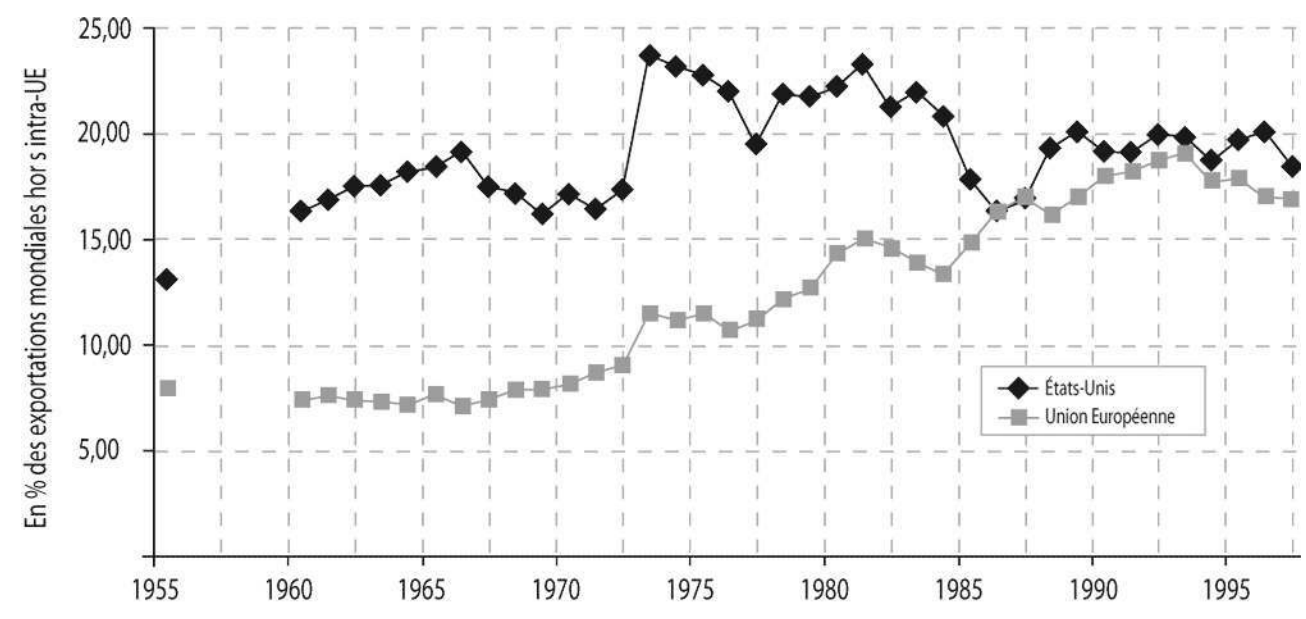

Source : Daviron et Voituriez (2006), d'après Cnuced et OMC

\section{Soyons égoïstes, soyez généreux}

Parmi les modifications apportées par l'OMC au GATT figure en bonne place l'élargissement des secteurs couverts par la négociation, celle-ci portant dorénavant sur les biens (y compris agricoles), les services et la propriété intellectuelle dans ses aspects relatifs au commerce. L'extension des compétences du GATT et le durcissement des obligations faites aux membres de l'OMC (limitation des possibilités de dérogation ou opt out, inversement de la règle d'unanimité lors du règlement des différends) peuvent se concevoir comme une réponse apportée à l'épuisement du système GATT. Celui-ci, en parvenant à abaisser les droits de douane industriels des pays à haut revenu à moins de $3 \%$ en moyenne au début des années 1990, n'avait pu empêcher un report de protection, soit sur d'autres secteurs (ainsi celui de l'agriculture), soit au moyen d'instruments non tarifaires (anti-dumping, restriction volontaires d'exportation), de mesures ad hoc de sauvegardes et d'accords plurilatéraux. Là où les antécédents récents de la guerre avaient donné au GATT, en 1948, une justification impérative et supplémentaire à celle d'une efficacité accrue des échanges, l'OMC, à sa naissance, en 1995, n'avait pour justifications que les deux autres « réponses» mentionnées plus haut, à savoir le contournement des lobbys protectionnistes et l'érosion des droits de douane des grands pays prisonniers de leur propre politique ${ }^{2}$. C'est en ce sens que l'on peut comprendre le durcissement des obligations, les lobbys ayant appris à manipuler le GATT. C'est ainsi également que l'on peut comprendre l'extension des domaines de négociation, puisque les protections entre grands pays ont certes été réduites pour l'essentiel des produits industriels mais subsistent malgré tout dans d'autres secteurs, agriculture et services en tête.

Le mandat de Doha, rédigé « les fesses serrées » quelques semaines après les attentats du 11 septembre 2001, vise à mettre le commerce au service du développement et rééquilibrer le précédent accord en faveur des pays non industrialisés. On pourrait penser, a posteriori, qu'il prolonge le mandat du GATT de faire contribuer le commerce à la paix, un facteur de conflit - ici de terreur -, étant non pas le protectionnisme généralisé 
mais les inégalités d'accès aux politiques et aux marchés séparant les pays riches des pays pauvres. Doha, alors, rétablirait l'impératif de paix quelque peu dissipé depuis 1947 et, plus encore, depuis 1989. La proximité de la ministérielle de Doha avec le 11 septembre ne permet pas toutefois de valider une telle lecture, la contribution, même indirecte, des inégalités de politique et d'accès aux inégalités de revenu, et de manière ultime, au terrorisme et à l'insécurité, n'ayant pu être l'objet d'un discours doctrinal construit et argumenté dans un si bref délai. Et voilà la libéralisation commerciale multilatérale promue facteur de développement, suggérant par là qu'elle ne l'était pas auparavant.

11 On a vu que le GATT puis l'OMC aidait chacun des pays membres à poursuivre son propre intérêt. Doha oblige chaque pays membre, et en particulier les plus riches, à faire en sorte que la poursuite de son propre intérêt ne contrevienne pas à l'engagement solennel d'obtenir, après accord, une répartition des gains de l'échange qui soit favorable aux pays en développement. Continuez d'être égoïste, dit l'OMC, nous pouvons vous y aider, mais soyez généreux. Premier problème, qui complique le mandat des négociateurs, puisque ceux-ci doivent rentrer chez eux avec un «bon accord " pour eux-mêmes et un bon accord pour les pays en développement. Que l'issue en soit un plus petit dénominateur commun n'est alors pas surprenant, l'accord épousant les limites des intérêts partagés. Un second problème, plus sérieux, ne manque pas de surgir : être généreux, très bien, mais être généreux pour qui ?

La question soulève plusieurs difficultés. La première, fort simple en apparence, est celle de la définition des critères sur laquelle les pays doivent s'entendre afin d'établir si l'accord est ou n'est pas bon pour le développement. Or, ni le GATT ni l'OMC, ne se sont sérieusement penchés sur la question, la meilleure preuve étant qu'aucune définition d'un "pays en développement» n'est contenue dans les différents textes de l'Organisation. Peut se prétendre en développement tout pays qui le décide, sauf si d'autre pays venaient à contester pareille affirmation. Est-il juste, par exemple, que la réforme de la PAC profite bien davantage aux pays émergents exportateurs qu'aux pays les moins avancés? Non, s'insurge d'une même voix le syndicalisme agricole français. Et de redoubler d'indignation en devinant que les premiers bénéficiaires d'un accès accru au marché européen pourraient être les riches propriétaires terriens brésiliens ou sud africains ${ }^{3}$.

13 - Une seconde difficulté, plus en amont, est celle de la conception de la justice que sousentend le terme de développement. Qu'est-ce qu'un accord juste? La question contenait sa propre réponse dans les principes et les modes de décision des précédents cycles de négociation. La réciprocité, d'une part, assure à tous les pays de recevoir au moins autant que ce qu'ils sont prêts à céder, l'unanimité permettant, d'autre part, à n'importe lequel d'entre eux de rejeter un accord jugé inéquitable. Justice procédurale donc, qui permettait d'aboutir à un accord acceptable par toutes les parties. Si l'on admet cette fois que certains doivent recevoir plus qu'ils ne peuvent donner, en supposant régler les difficultés de définition et d'identification des pays bénéficiant d'un surcroît d'avantages dans le partage des gains de l'échange, demeure l'ardu problème du montant complémentaire (ou de son équivalent en terme d'accès au marché) qu'il est juste de concéder à chacun, et à défaut, des principes qui permettront d'obtenir un tel montant de manière concertée et consensuelle. Justice procédurale, toujours. En l'absence d'un tel principe, l'offre américaine de garantir un accès au marché libre de droit de douane et de quota à au moins $97 \%$ des produits originaires des pays les moins avancés avant 2008, passer paraîtra par exemple une largesse à certains et une mesquinerie à d'autres qui 
allègueront que les $3 \%$ concernent des produits clefs comme certains produits textiles. Dès lors, si à cause du "développement", la réciprocité semble un principe frappé de caducité, un autre principe doit lui être substitué, qui reste à identifier ${ }^{4}$.

Qu'est-ce qu'un accord juste demandions-nous ? D'un point de vue substantiel, les réponses que l'on rencontre ne sont ni plus claires ni plus consensuelles qu'en matière procédurale. Un accord juste signifie quantité de choses aujourd'hui. Ainsi, corrige-t-il des injustices passées, affirment les Brésiliens, qui au nom de cette définition refusent d'ouvrir leurs marchés aux produits industriels européens avant que l'Europe n'ait consenti à leur ouvrir en grand l'accès à son marché agricole, accès qui, au nom de cette définition intertemporelle de la justice leur serait dû. Un accord juste, dans un sens rawlsien très approximatif, améliore le sort des plus défavorisés, prétendront d'autres. Las, que nous enseignent les travaux empiriques sur les liens entre ouverture commerciale, pauvreté et inégalités? Une foule de choses, parfois contradictoires, mais dont il semble ressortir malgré tout que la belle histoire racontée par Hecksher et Ohlin selon laquelle, en augmentant la demande de main d'oeuvre non qualifiée relativement abondante, la libéralisation commerciale allait, dans les pays en développement, contribuer à réduire les inégalités. Cette belle histoire ne s'observe pas, si l'on s'en tient aux inégalités de salaire à tout le moins (Chabe-Ferret, Gourdon, Marouani, Voituriez, 2006). Il n'existe donc pas de mesure de libéralisation particulière qui, réglant son sort à la pauvreté à tout coup, pourrait être retenue comme priorité de l'agenda de négociation ${ }^{5}$

L'ultime difficulté enfin, en miroir de la première, concerne la répartition des efforts en faveur des pays en développement. Parce que chaque pays sait ce qu'il gagne mais ignore le gain véritable obtenu par les autres pays, l'obtention d'une répartition juste des efforts est un concours d'obstacles tout au long duquel chacun proclame, vainement, la suprématie de sa générosité.

\section{Les pays émergents ont à présent plus à perdre qu'à gagner}

16 Cependant qu'à Cancun, en 2003, l'absence d'accord commercial avait été jugée préférable à un mauvais accord - en particulier pour les pays en développement - à Hong-Kong la situation a été exactement inverse. Une apparence d'accord était à l'unanimité préférable à la rupture des négociations. Les causes de ce revirement se trouvent dans le poids politique considérable pris depuis Cancun par les pays émergents et en particulier par le Brésil. Ce sont les pays émergents qui ont pris le risque de porter la responsabilité d'un soi disant « échec » à Cancun. Ce sont les pays émergents - Brésil et Inde - qui, à côté des EU, de l'UE et de l'Australie ont formé le « FIP » (five interested parties) qui a pris l'initiative de rédiger l'accord-cadre de juillet 2004 sur la base duquel s'est déroulé Hong-Kong. Ce sont encore les pays émergents qui, au sein du FIP, ont contraint l'Europe et les États-Unis à préciser jusqu'aux toutes dernières semaines avant Hong-Kong, les modalités d'abaissement des tarifs et des soutiens agricoles. Et c'est parce qu'ils ont, même imparfaitement, eu gain de cause que les pays émergents se trouvaient à l'ouverture de la ministérielle devant cette situation inédite d'avoir plus à perdre qu'à gagner, même si l'Inde, par l'intermédiaire de son ministre du commerce, continue de marteler « no deal is better than a bad deal» dans une rhétorique dont on peut douter qu'elle corresponde aux intérêts réels des autres grands pays émergents et du Brésil en particulier. 
Il est certain désormais que les accords à l'OMC ne seront plus pré-rédigés par les ÉtatsUnis et l'Europe ce qui place l'OMC et les pays émergents devant des difficultés nouvelles. À l'OMC de trouver un modus operandi capable d'intégrer des " parties intéressées » aux intérêts économiques ouvertement divergents (à tout le moins en matière agricole). Au Brésil, à la tête des pays émergents, " grand pays " importateur et exportateur, de prendre l'initiative d'un accord favorable à toutes les parties intéressées, y compris aux États-Unis et au vieux continent, démontrant par là même l'élévation de sa stature à celle d'un grand pays des relations internationales - et n'ont plus seulement à celle d'un grand pays commercial, que nul ne lui conteste plus maintenant.

\section{BIBLIOGRAPHIE}

Bagwell K., Steiger R.-W. (1999). An Economic Theory of Gatt. American Economic Review, vol. 89, $n^{\circ} 1$, p. 215-248.

Chabe-Ferret S., Gourdon J., Marouani M.-A., Voituriez T. (2006). Trade-Induced Inequalities: Assessment Issues and Policy Implications for Developing Countries. Tokyo, Communication à la Conférence ABCDE, Banque Mondiale, 29-30 mai.

Daviron B., Voituriez T. (2006). Quelle régulation des échanges agricoles internationaux ? Un éclairage par la théorie des régimes. In Berthaud P., Kébadjan G. « La question politique en économie internationale ", La Découverte.

Hertel T., Winters A.-L. (2006). Poverty and the WTO. Impacts of the Doha Development Agenda. Washington DC and Palgrave Macmillan, The World Bank.

Hoekman B.-M., Kostecki M.-M. (2001). The Political Economy of the World Trading System. Oxford University Press.

Irwin D. (2002). Free Trade Under Fire. Princeton University Press.

Pastor R. (1983). The cry-and-sigh syndrom, Congress and trade policy. In Allen Shick ed. "Making Economic Policy In Congress", Washington D.-C., American Enterprise Institute.

Roessler F. (1985). The Scope, Functions and Limits of the World Trading System. The World Economy, n8, p. 289-298.

Stiglitz J., Charlton A. (2006). Fair Trade for All. How Trade Can Promote Development. Oxford University Press.

\section{NOTES}

1. Mettant en garde contre les conséquences économiques désastreuses des droits de douane proposés par les deux élus républicains, 1028 économistes signèrent une pétition enjoignant le Président Hoover de ne pas souscrire au vote du Congrès. En 1933, le droit de douane moyen sur les importations (frappées d'un droit de douane, ou « dutiable imports ») était de $54 \%$, soit près du 
double de celui de 1925. Les exportations et importations chutèrent de $50 \%$ entre 1929 et 1933 (Irwin, 2002).

2. On pourra toujours objecter que l'objectif ultime de "développement durable ", ajouté au préambule du GATT de 1994, visait à se substituer à l'objectif de paix que l'on trouve en filigrane du GATT de 1947. Reste que la contribution du commerce au développement durable manque de l'autorité d'un Montesquieu qui, sauf erreur de notre part ne s'est pas prononcé sur le sujet, se bornant à affirmer que « l'effet naturel du commerce est de porter à la paix ».

3. La Banque Mondiale, d'une efficacité redoutable, ne tarda pas à démolir l'argument, estimant (au sens propre du terme), que les salariés in fine en profitaient par un accroissement de leur salaire (Hertel et Winters, 2006).

4. Des propositions existent, ainsi Stiglitz et Charlton (2005) proposent une règle procédurale de justice selon laquelle chaque pays assure à tous les pays plus petits (PIB) et plus pauvres (PIB/ capita) que lui d'avoir un accès complet à son marché.

5. L'accès au marché des pays riches répond néanmoins au plus près à ce critère, raison pour laquelle, sans doute, il est réclamé en priorité par l'écrasante majorité des pays en développement.

\section{AUTEUR}

\section{TANCRÈDE VOITURIEZ}

Cirad, chercheur associé à l'Iddri 